ACCEPted For Publication in ApJ LetTers

Preprint typeset using LATEX style emulateapj v. 08/22/09

\title{
A PILOT SURVEY OF HI IN FIELD GALAXIES AT REDSHIFT Z 0.2
}

\author{
Barbara Catinella ${ }^{1,2}$, Martha P. Haynes ${ }^{3}$, Riccardo Giovanelli ${ }^{3}$, Jeffrey P. Gardner ${ }^{4}$, \& Andrew J. Connolly ${ }^{5}$ \\ Accepted for publication in ApJ Letters
}

\begin{abstract}
We present the first results of a targeted survey carried out with the $305 \mathrm{~m}$ Arecibo telescope to detect HI-line emission from galaxies at redshift $z>0.16$. The targets, selected from the Sloan Digital Sky Survey database, are non-interacting disk galaxies in relatively isolated fields. We present here the Hi spectra and derived Hi parameters for ten objects detected in this pilot program. All are massive disk galaxies in the redshift interval 0.17-0.25 (i.e. 2-3 Gyr look-back time), with Hi masses $M_{\mathrm{HI}}=3-8 \times 10^{10} \mathrm{M}_{\odot}$ and high gas mass fractions (HI- to - stellar mass ratios $\sim 10-30 \%$ ). Our results demonstrate the efficacy of exploiting Arecibo's large collecting area to measure the Hi mass and rotational velocity of galaxies above redshift $z=0.2$. In particular, this sample includes the highest redshift detections of Hi emission from individual galaxies made to date. Extension of this pilot program will allow us to study the Hi properties of field galaxies at cosmological distances, thus complementing ongoing radio synthesis observations of cluster samples at $z \sim 0.2$.

Subject headings: galaxies: evolution — galaxies: spiral — radio lines: galaxies — cosmology: observations
\end{abstract}

\section{INTRODUCTION}

Because cold atomic hydrogen represents the reservoir for future star formation and the physics of the $21 \mathrm{~cm}$ transition is relatively simple, detection of the Hi line emission has the potential to contribute a key quantitative ingredient to models of how galaxies acquire and retain gas and convert it into stars. Of critical importance is the tracking of the gas content over the redshift interval between $z \sim 1$ and 0, i.e. over the period when the observed cosmic star formation rate density has declined by an order of magnitude (e.g., Lilly et al. 1996; Madau et al. 1996; Bell et al. 2005, and references therein). However, our knowledge of the Hi $21 \mathrm{~cm}$ line emission properties of galaxies is currently limited to the very local universe, and only recently have technical improvements in radio frequency receivers and back-ends made attempts to explore the Hi emission from galaxies at $z>0.1$ in any practical sense.

The first detection of HI emission from a galaxy at cosmological distance $(z=0.1766)$ was made by Zwaan, van Dokkum, \& Verheijen (2001) with the Westerbork Synthesis Radio Telescope (WSRT). More recently, Verheijen et al. (2007) successfully carried out a pilot program with the WSRT to image $z \sim 0.2$ galaxy clusters at $21 \mathrm{~cm}$. They targeted the Abell 963 $(z=0.206)$ and Abell $2192(z=0.188)$ clusters for

\footnotetext{
${ }^{1}$ Max-Planck-Institut für Astrophysik, D-85741 Garching, Germany; bcatinel@mpa-garching.mpg.de.

2 Arecibo Observatory, HC3 Box 53995, Arecibo, PR 00612, USA. The Arecibo Observatory is part of the National Astronomy and Ionosphere Center, which is operated by Cornell University under a cooperative agreement with the National Science Foundation.

${ }^{3}$ Center for Radiophysics and Space Research and National Astronomy and Ionosphere Center, Cornell University, Ithaca, NY 14853, USA.

${ }^{4}$ Department of Physics, University of Washington, Seattle, WA 98195, USA.

${ }^{5}$ Department of Astronomy, University of Washington, Seattle, WA 98195, USA.
}

$20 \times 12$ hours and $15 \times 12$ hours respectively, and detected Hi emission from 42 galaxies. The Giant Metrewave Radio Telescope was used by Lah et al. (2007) to constrain the Hi content of star forming galaxies at $z=0.24$. They obtained an average Hi spectrum by co-adding the nondetections of 121 galaxies with known optical redshifts. However, their detection is at best marginal.

Historically, Arecibo's huge collecting area has offered a critical advantage to Hi line studies of extragalactic objects, because sensitivity is of paramount importance to the detection of weak Hi signals. Recent improvements to the Arecibo instrumentation, both front-end and back-end, are allowing the practical exploration of the frequency range corresponding to HI at $z>0.1$. Most notably, the installation of the new, single-pixel L-band receiver ("L-wide") in February 2003 has given access to frequencies down to $1120 \mathrm{MHz}$ (corresponding to redshifted Hi emission at $z=0.27$ ) with improved system noise and performance. Naturally, single-dish Hi observations of intermediate redshift galaxies are challenged by a lack of angular resolution. As the density of galaxies per unit solid angle increases with redshift, so also does the probability of finding multiple sources within the telescope beam. However, beam confusion can be broken kinematically, if high-quality optical redshifts are available for the sources within the beam. The availability of imaging and spectroscopic data from the Sloan Digital Sky Survey (SDSS; York et al. 2000) has enabled the identification of sources suitable for long-integration HI line observations with the Arecibo telescope. In this Letter, we report the first results of a pilot survey carried out at Arecibo to detect Hi emission from disk galaxies at $z \sim 0.2$.

All the distance-dependent quantities in this work are calculated assuming $H_{0}=71 \mathrm{~km} \mathrm{~s}^{-1} \mathrm{Mpc}^{-1}, \Omega_{\mathrm{M}}=0.27$ and $\Omega_{\mathrm{vac}}=0.73$. 


\section{SAMPLE, OBSERVATIONS AND DATA REDUCTION}

The initial goal of this survey was to explore the feasibility of detecting Hi line emission from galaxies beyond the redshift range of past Arecibo surveys, that is at redshifts $z>0.05$. First observations successfully detected over 50 galaxies with $0.05 \leq z \leq 0.09$, and the program was extended to higher redshifts with the availability of the new L-wide receiver. In the end, the frequency interval corresponding to $0.11<z<0.16$ was excluded because of the particularly hostile Radio Frequency Interference (RFI) environment. Here, we report results only for objects with $z>0.16$; the full dataset will be presented elsewhere (B. Catinella et al., in preparation).

The targets for HI spectroscopy were extracted from the SDSS database according to the following criteria: (a) observable from Arecibo during night-time (to minimize RFI); (b) redshift $0.16<z<0.27$ and not corresponding to the frequency of known RFI within that interval. This redshift range corresponds to the 1120-1220 $\mathrm{MHz}$ frequency interval and is dictated by the instrumentation (see below); (c) inclination $i \geq 45^{\circ}$ (for use in disk scaling relations); (d) presence of $\mathrm{H} \alpha$ emission with line width between 100 and $700 \mathrm{~km} \mathrm{~s}^{-1}$ and equivalent width larger than $5 \AA$; (e) exponential disk profile; (f) noninteracting, undisturbed, disk appearance in the SDSS images. The SDSS images of the candidate targets were carefully inspected to exclude objects with interacting or peculiar appearance, and/or with Hi emission possibly contaminated by that of other, nearby objects lying within the Arecibo beam. We also discarded targets lying in the vicinity of continuum sources that would cause ripples in the baselines.

The Hi data for the $z>0.16$ sample were collected during four observing runs, distributed between Fall 2003 and Spring 2007. Including all the overheads, the total time spent on this part of the survey was 280 hours. The observations were conducted in standard positionswitching mode: each observation consisted of an on/off source pair, each integrated for 4 minutes $(5$ minutes in the last observing run), followed by the firing of a calibration noise diode. The off observation of a "blank sky" region was performed by tracking exactly the same range of azimuth and zenith angle as was previously occupied by the on source, as commonly done at Arecibo to optimize the bandpass subtraction. The on-source integration time per object varied between 80 minutes and 4.7 hours, primarily depending on the redshift and on the observed rotational velocity of the target (for the same Hi flux, slower rotators are easier to detect). The spectra were acquired using the L-band wide receiver, which operates in the frequency range $1120-1730 \mathrm{MHz}$. We also used a $1120-1220 \mathrm{MHz}$ filter and a $750 \mathrm{MHz}$ narrow band front-end filter to limit the impact of RFI on our observations. Although sensitivity requirements suggest long accumulations of data, RFI considerations dictate fast spectral dumping, because much RFI is of short duration. Thus spectra were recorded every second with 9-level sampling. Two correlator boards, each configured for $12.5 \mathrm{MHz}$ bandwidth, one polarization, and 2048 channels per spectrum (yielding a velocity resolution of $1.8 \mathrm{~km} \mathrm{~s}^{-1}$ at $1200 \mathrm{MHz}$ before smoothing) were centered at or near the frequency corresponding to the SDSS redshift of the target. Minimizing the number of different board settings facilitates the identification of RFI. In order to better discriminate terrestrial sources from cosmic ones, we also chose not to apply any online Doppler correction for the motion of the Earth (the correction is applied during off-line processing).

The data reduction was performed using our own routines based on the standard Arecibo data processing library and will be described in full detail elsewhere. In summary, the data processing of each polarization and on-off pair includes the following steps: Hanning smoothing, bandpass subtraction, RFI excision, and flux calibration. A final spectrum for each of the two orthogonal linear polarizations for each galaxy is constructed by the combination of good quality records (those without serious RFI or standing waves), weighting each by a factor $1 / r m s^{2}$, where rms is the root mean square noise measured in the signal-free portion of the spectrum. The two polarizations are then averaged, yielding the final spectrum. After boxcar smoothing and baseline subtraction, the Hi-line profiles are ready for the measurement of redshift, rotational velocity and integrated Hi line flux. Our measurement technique is explained, e.g., in Catinella. Havnes, \& Giovanelli (2007, §2.2).

\section{RESULTS}

The total number of galaxies targeted by our $z>0.16$ pilot survey is 41 (this number does not include several other objects that were initially targeted but had to be abandoned after short integrations because of RFI or other problems). Half of the targeted galaxies are sure detections with high-quality Hi profiles, a subset of which is presented in this Letter. Of the remaining half, only $\sim 1 / 3$ are clearly non-detections; the remaining objects include marginal detections, uncertain profiles or otherwise problematic cases that are still being analyzed.

Figure1(Plate 1) presents 10 of the best quality Hi detections of $z \sim 0.2$ galaxies obtained at Arecibo, ordered by increasing redshift (as indicated next to each spectrum). These galaxies are not necessarily the highest signal-to-noise detections, but have been chosen to span the entire $0.16<z<0.25$ redshift interval covered by our pilot program. Each panel of Fig. 1 shows the SDSS image $\left(1^{\prime}\right.$ size, which is equivalent to about $1 / 4$ of the half power full width of the Arecibo beam at $1200 \mathrm{MHz}$ ) and the Hi spectrum of the targeted galaxy (the full 12.5 $\mathrm{MHz}$ bandwidth is shown). The Hi-line profiles are boxcar smoothed by 15 channels, corresponding to a velocity resolution of $27 \mathrm{~km} \mathrm{~s}^{-1}$ at $1200 \mathrm{MHz}$, and baselinesubtracted. The spectra are labeled with two numbers, the Arecibo General Catalog (AGC; a private database maintained at Cornell University by MPH and RG) identifier and the SDSS redshift of the galaxy. The frequency and heliocentric velocity (bottom and top $x$ axis, respectively) corresponding to the SDSS redshift are indicated by a dashed line. The dotted line is at the redshift measured from the HI spectrum. The last three objects, with $z>0.21$, represent the highest redshift detections of HIline emission from individual galaxies made to date.

Table 1 lists SDSS and Hi parameters for the galaxies presented in Fig. 1, similarly ordered by increasing redshift. The quantities are listed as follows: Columns (1) and (2) are the source identifiers in the AGC and SDSS databases. Column (3) is the SDSS redshift, $z_{\mathrm{SDSS}}$; the associated uncertainty on the last digit is shown within 
brackets. Column (4) is the absolute $I$-band magnitude in the Cousins system, $M_{\mathrm{I}}$ (see $\S 3.1$ ). Column (5) is the galaxy inclination to the line-of-sight in degrees, $i$, estimated from SDSS tabulated axis ratios in $r$-band. Column (6) is the on-source integration time of the Arecibo observation, $T_{\text {on }}$, in minutes (only on scans that were actually combined are counted). Column (7) is the redshift, $z$, measured from the Hi spectrum. The error on the corresponding heliocentric velocity, $c z$, is half the error on the width, tabulated in the following column. Column (8) is the observed velocity width of the source line profile in $\mathrm{km} \mathrm{s}^{-1}, W_{50}$ (measured at the $50 \%$ of the peak levels), with its statistical uncertainty. Column (9) is the velocity width corrected for instrumental broadening and cosmological redshift only, $W_{50}^{c}$. No inclination or turbulent motion corrections are applied. Column (10) is the observed, integrated $\mathrm{HI}$-line flux density in $\mathrm{Jy} \mathrm{km} \mathrm{s}^{-1}$, $F \equiv \int S d v$, measured on the smoothed and baselinesubtracted spectrum. The statistical errors are calculated according to equation 2 of Springob et al. (2005, hereafter S05). Column (11) is the signal-to-noise ratio of the Hi spectrum, S/N, estimated following Saintonge (2007) and adapted to the velocity resolution of our spectra. This definition of $\mathrm{S} / \mathrm{N}$ accounts for the fact that for the same peak flux a broader spectrum has more signal. Column (12) is the base-10 logarithm of the Hi mass, $M_{\mathrm{HI}}$, in solar units. The HI masses of these galaxies vary between 3 and $8 \times 10^{10} \mathrm{M}_{\odot}$ and were computed via:

$$
\frac{M_{\mathrm{HI}}}{\mathrm{M}_{\odot}}=\frac{2.356 \times 10^{5}}{1+z}\left[\frac{d_{\mathrm{L}}(z)}{\mathrm{Mpc}}\right]^{2}\left(\frac{\int S d v}{\mathrm{Jy} \mathrm{km} \mathrm{s}^{-1}}\right)
$$

where $d_{\mathrm{L}}(z)$ is the luminosity distance to the galaxy at redshift $z$.

As both Figure 1 and Table 1 demonstrate, there is an excellent agreement between SDSS and Hi redshifts (the largest discrepancy is $\Delta z=0.0004$ for AGC 252580 and AGC 224321. However, the difference drops to $\Delta z=0.0001$ for AGC 224321 when the SDSS emission line redshift ${ }^{6}$ is used instead. The emission line redshift of AGC 212941, 0.2238, is also in slightly better agreement with the Hi one). The importance of having accurate SDSS redshifts is exemplified by AGC 242147, the highest redshift detection, whose spectrum shows strong RFI at $1137 \mathrm{MHz}$. Because of the long integration required and because in the first hour or two of observation one can see the RFI but not the galaxy's line emission, it is essential to know in advance precisely where the $\mathrm{HI}$ signal is expected to appear.

In $\S 2$ we noted that, at fixed redshift and Hi flux, galaxies that appear to be slower rotators (i.e., either intrinsically slower or just viewed more face-on) are significantly easier to detect. This point is clearly illustrated by the two galaxies shown in the second row of Fig. 1] AGC 262033 and AGC 224321, which have among the largest and the smallest observed velocity widths in this sample, respectively. These are also the objects that required the longest and the shortest integrations, respectively, in spite of having almost the same redshift and Hi flux.

The rotational velocities deprojected to edge-on view (i.e., $0.5 W_{50}^{c} / \sin i$ ) vary between $\sim 180$ and $320 \mathrm{~km} \mathrm{~s}^{-1}$ for this sample. These values are consistent with those

\footnotetext{
6 Not available for AGC 252580.
}

typical of local disk galaxies with similar rest-frame $I$ band luminosities, as shown for a large set of $\mathrm{H} \alpha$ rotation curves by Catinella, Giovanelli, \& Havnes (2006, Fig. 1), also taking into account that optical velocity widths tend to be systematically smaller than $21 \mathrm{~cm}$ ones (a bias understandable in terms of rotation curve shapes and relative extent of Hi vs. $\mathrm{H} \alpha$ emission; see, e.g., Catinella, Haynes, \& Giovanelli 2007).

We computed gas mass fractions, $M_{\mathrm{HI}} / M_{\text {star }}$, for the galaxies in our sample. Stellar masses, $M_{\text {star }}$, were estimated from SDSS $i$-band model magnitudes and $g-i$ colors, using Bell et al. (2003) stellar mass-to-light vs. color correlations ${ }^{7}$. We corrected the SDSS magnitudes for Galactic attenuation first, and applied Springob et al. (2007, hereafter S07) K- and internal extinction corrections (the extinction in SDSS $g, i$ bands was obtained from that in S07 Cousins $I$ assuming a standard attenuation curve of the form $\left.\tau_{\lambda} \propto \lambda^{-0.7}\right)$. The objects detected at Arecibo are very massive, gas-rich galaxies, with gas mass fractions $M_{\mathrm{HI}} / M_{\text {star }}$ of $\sim 10-30 \%$, reflective of the deliberate selection of galaxies on the basis of their potentially high Hi content.

\subsection{Comparison with $z=0$ field galaxies}

Since $z \sim 0.2$ corresponds to a non-negligible fraction of the age of the universe, and in particular, the epoch over which the star formation density has rapidly declined, it is interesting to investigate if this small sample is already suggestive of possible evolutionary effects. This exercise requires a representative comparison data set of field galaxies at $z=0$ which we have extracted from the Cornell Hi digital archive (S05). In addition to being a high-quality, homogeneous compilation of Hi parameters for a large number of galaxies, a subset of the objects in the Hi archive has matching I-band photometry and grouping information from the SFI++ catalog (S07). The cross-match of the S05 Hi archive (8850 objects) with the S07 photometry (Table 2: 4053 objects, 2713 of which not listed as group members), after rejection of galaxies with recessional velocities $c z>10000$ $\mathrm{km} \mathrm{s}^{-1}$, includes 1081 objects. The redshift cut was applied in order to obtain a clean $z=0$ sample, but the inclusion of the 124 galaxies with $c z>10000 \mathrm{~km} \mathrm{~s}^{-1}$ (the largest recessional velocity is $17897 \mathrm{~km} \mathrm{~s}^{-1}$ ) would not change any of our conclusions. Since color information is not available for the $\mathrm{SFI}++$ galaxies, we could not estimate stellar masses. Thus we compared Hi mass to $I-$ band luminosity ratios, $M_{\mathrm{HI}} / L_{\mathrm{I}}$, for the two samples. We used standard transformations ${ }^{8}$ to convert SDSS model $r$ magnitudes into Cousins $I$ for our sample, and rescaled the SFI++ quantities to our adopted Hubble constant. The resulting $M_{\mathrm{HI}} / L_{\mathrm{I}}$ ratios for our data set (large circles) and for the $z=0$ reference sample (small symbols) are presented in Fig. 2. Dotted lines indicate lines of constant HI mass, $M_{\mathrm{HI}}=2,4,8 \times 10^{10} \mathrm{M}_{\odot}$ (bottom to top, respectively). The reference sample has a very large scatter, and contains only a small number of galaxies

7 As Bell et al. (2003) note, their choice of stellar initial mass function yields "the maximum possible stellar $M / L$ ratio". Thus, at given luminosity, we might overestimate the stellar masses and therefore underestimate the gas mass fractions.

8 See http://www.sdss.org/dr6/algorithms/sdssUBVRITransform.html (Lupton 2005 equations). 
with rest-frame luminosities comparable with those of our sample. This limitation makes it very difficult to estimate where the center of the distribution might be, i.e. what is the "typical" HI mass of a local field galaxy with $L_{\mathrm{I}} \sim 10^{11} \mathrm{~L}_{\odot}$. The objects in our sample are, on average, very gas-rich, and lie on or slightly above the upper envelope of the local galaxy distribution. Naturally, this selection effect arises because we detect only the gas-richest galaxies at $z=0.2$. Admittedly, galaxies with such large $M_{\mathrm{HI}} / L_{\mathrm{I}}$ ratios at these luminosities do appear to be rare in the local universe. However, since our survey probes a much larger volume compared to the $z=0$ sample, it is perhaps not surprising to detect several of these locally rare objects.

Understanding and quantifying evolutionary effects requires the use of complete samples with well-defined selection criteria. Clearly, this is not the case for our current survey, which was in part a proof-of-feasibility experiment. Unfortunately, the $z=0$ reference sample is not complete in any sense, either. In particular, it is currently very difficult to find local Hi samples with large numbers of galaxies that are as massive and/or as HIrich as our detections. Thus, we cannot make any strong statements about the evolution of the Hi mass-to-light ratio based on the results shown in Fig. 2 .

\section{CONCLUSIONS}

In this Letter, we have shown that carefully-planned Arecibo observations can deliver high-quality global Hi profiles for galaxies at redshifts up to $z=0.25$ with integration times of a few hours per object. The detected objects are massive disk galaxies with high gas mass fractions $(\sim 10-30 \%)$, and are apparently rare in the local universe. At present, the small size of our sample, selection effects and the lack of adequate comparison samples at $z=0$ prevent us from drawing any firm conclusions about the evolution of the $\mathrm{HI}$ content of galaxies between redshifts 0.25 and 0 . Larger and well-defined samples of galaxies at $z \sim 0.2$ and higher are needed in order to make progress in this area. Equally importantly, fair comparison samples at $z=0$ are also needed. The latter might be provided by two large surveys that are ongoing at Arecibo. The first one is the Arecibo Legacy Fast ALFA (ALFALFA) survey (Giovanelli et al. 2005, 2007), a blind Hi survey of the extragalactic sky visible from Arecibo, which will make a complete census of Hi-rich galaxies with $z \leq 0.060$ and has already detected a significant population of objects with Hi masses $>10^{10} \mathrm{M}_{\odot}$ (Haynes 2008). The second one is the GALEX Arecibo SDSS Survey (GASS; Catinella, Schiminovich, \& Kauffmann 2008), a recently started program designed to measure the Hi content of $\sim 1000$ massive galaxies, randomly selected from the intersection of the SDSS spectroscopic survey, GALEX and ALFALFA footprints based on their redshift $(0.025<z<0.05)$ and stellar mass $\left(10.0<\log \left[M_{\text {star }} / \mathrm{M}_{\odot}\right]<11.5\right)$ only. ALFALFA and GASS will deliver unbiased samples of local HI-rich and massive galaxies, respectively. In combination with the future extension of our pilot intermediate redshift field galaxy program and with complementary Hi synthesis mapping programs of similar galaxies in crowded cluster and group fields, it is clearly now feasible to explore the evolution of the Hi content of disk galaxies over the last several Gyr for a statistically complete sample.

BC wishes to thank the Arecibo staff, in particular Ganesan Rajagopalan and the telescope operators for their assistance, and Hector Hernandez for scheduling the observations. Many special thanks to Phil Perillat for his invaluable help on data processing and calibration issues. Becky Koopmann and Lisa Wei helped getting the data reduction pipeline started in the early stages of the survey. BC also thanks Luca Cortese for useful discussions and Guinevere Kauffmann for helpful comments on the manuscript. We thank an anonymous referee for constructive feedback. This research has made use of the Sloan Digital Sky Survey archive. Its full acknowledgment can be found at http://www.sdss.org. We acknowledge the use of the NASA/IPAC Extragalactic Database (NED), which is operated by the Jet Propulsion Laboratory, California Institute of Technology, under contract with NASA. This work has been partially supported by NSF grants AST-0307396 and AST-0307661 and by NAIC.

\section{Facility: Arecibo (L-wide)}

\section{REFERENCES}

Bell, E. F., McIntosh, D. H., Katz, N., \& Weinberg, M. D. 2003, ApJS, 149, 289

Bell, E. F. et al. 2005, ApJ, 625, 23

Catinella, B., Giovanelli, R., \& Haynes, M. P. 2006, ApJ, 640, 751

Catinella, B., Haynes, M. P., \& Giovanelli, R. 2007, AJ, 134, 334

Catinella, B., Schiminovich, D., \& Kauffmann, G. 2008, to appear in AIP Conference Proceedings, The Evolution of Galaxies through the Neutral Hydrogen Window, eds. R. Minchin \& E. Momjian (arXiv:0803.4274)

Giovanelli, R. et al. 2005, AJ, 130, 2598

Giovanelli, R. et al. 2007, AJ, 133, 2569

Haynes, M.P. 2008, Il Nuovo Cimento (online available)

Lah, P. et al. 2007, MNRAS, 376, 1357

Lilly, S. J., Le Fèvre, O., Hammer, F., \& Crampton, D. 1996,

ApJ, 460, L1
Madau, P., Ferguson, H. C., Dickinson, M. E., Giavalisco, M., Steidel, C. C., \& Fruchter, A. 1996, MNRAS, 283, 1388 Saintonge, A. 2007, AJ, 133, 2087

Springob, C. M., Haynes, M. P., Giovanelli, R., \& Kent, B. R. 2005, ApJS, 160, 149 (S05)

Springob, C. M., Masters, K. L., Haynes, M. P., Giovanelli, R., \& Marinoni, C. 2007, ApJS, 172, 599 (S07)

Verheijen, M., van Gorkom, J. H., Szomoru, A., Dwarakanath, K. S., Poggianti, B. M., \& Schiminovich, D. 2007, ApJ, 668, L9 York, D. G., et al. 2000, AJ, 120, 1579

Zwaan, M. A., van Dokkum, P. G., \& Verheijen, M. A. W. 2001, Science, 293, 1800 
A Pilot Survey of HI in Field Galaxies at z 0.2
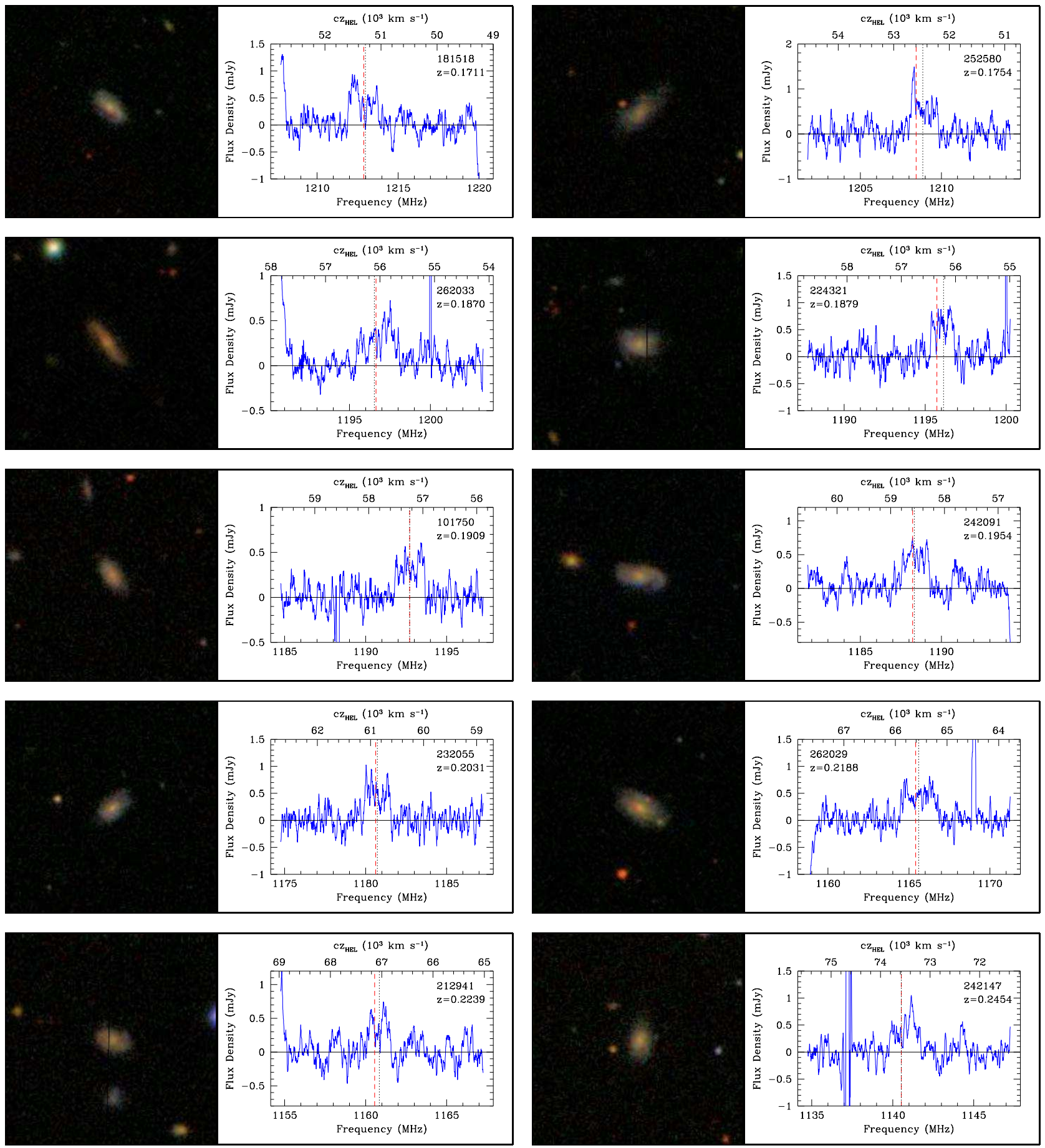

FIG. 1. - SDSS postage stamp images and Hi spectra of the 10 Arecibo detections discussed in this work. Each SDSS image is 1 arcmin square. The HI spectra are calibrated, smoothed and baseline-subtracted. Each spectrum is labeled with its AGC identifier and SDSS redshift. The central heliocentric velocity (frequency) corresponding to the SDSS redshift and that measured from the spectrum are indicated with a dashed and a dotted line, respectively. The $r m s$ noise per channel at the final velocity resolution $\left(27 \mathrm{~km} \mathrm{~s} \mathrm{~s}^{-1}\right.$ at 1200 $\mathrm{MHz}$ ), measured in the RFI-free portions of the baseline, varies between 0.1 and $0.2 \mathrm{mJy}$ for these spectra. 


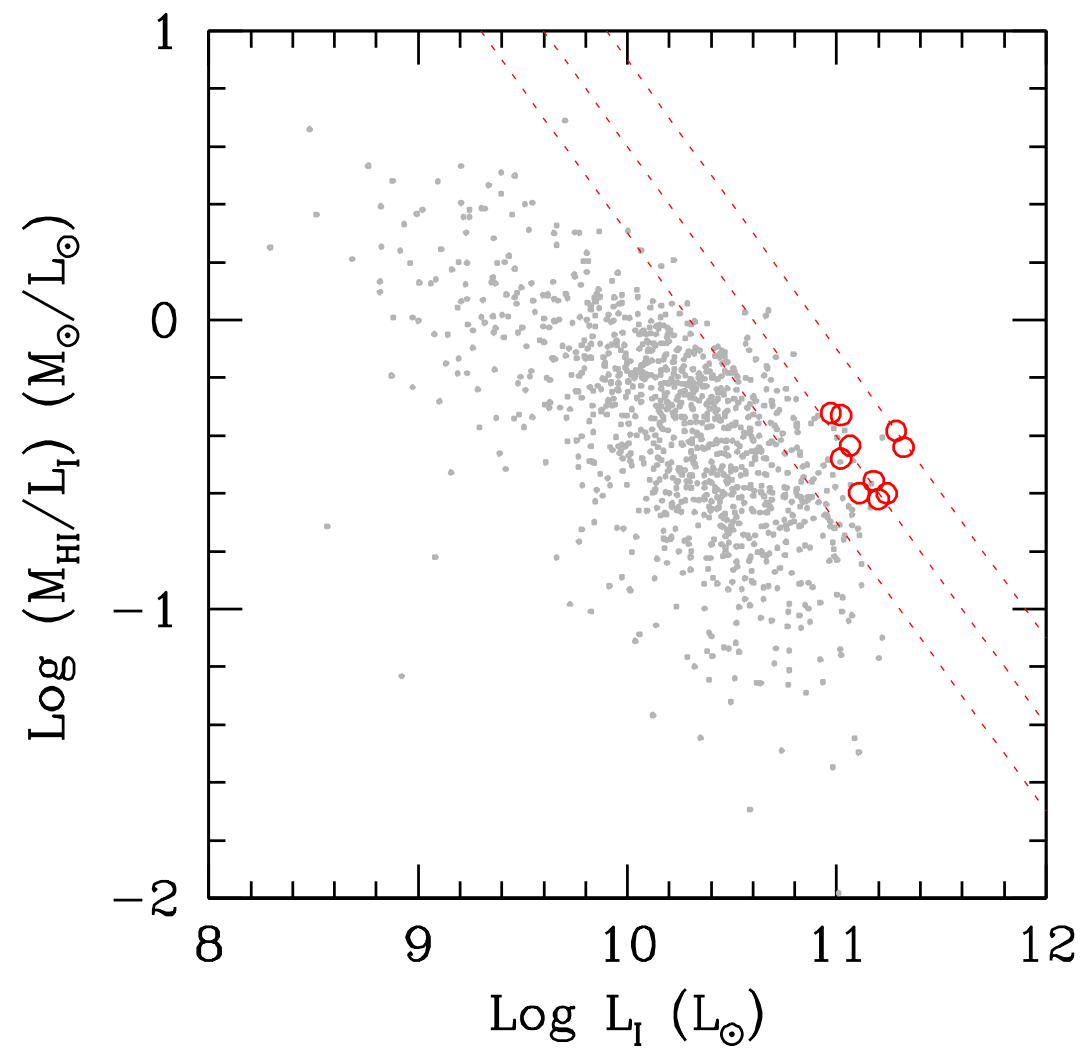

FIG. 2.- Hi mass to $I$-band luminosity ratio as a function of $I$-band luminosity for the Arecibo Hi detections (large circles) and for a comparison field sample at $z=0$ (small symbols). Dotted lines correspond to constant Hi mass, $M_{\mathrm{HI}}=2,4,8 \times 10^{10} \mathrm{M}_{\odot}$ (bottom to top, respectively).

TABLE 1

SDSS and Hi Parameters of the Arecibo Detections

\begin{tabular}{|c|c|c|c|c|c|c|c|c|c|c|c|}
\hline $\begin{array}{c}\mathrm{AGC} \\
(1)\end{array}$ & $\begin{array}{c}\text { SDSS ID } \\
(2)\end{array}$ & $\begin{array}{c}z_{\text {SDSS }} \\
(3)\end{array}$ & $\begin{array}{c}M_{\mathrm{I}} \\
(\mathrm{mag}) \\
(4)\end{array}$ & $\begin{array}{c}i \\
(\mathrm{deg}) \\
(5)\end{array}$ & $\begin{array}{c}T_{\text {on }} \\
(\min ) \\
(6)\end{array}$ & $\begin{array}{c}z \\
(7)\end{array}$ & $\begin{array}{c}W_{50} \\
\left(\mathrm{~km} \mathrm{~s}^{-1}\right) \\
(8)\end{array}$ & $\begin{array}{c}W_{50}^{c} \\
\left(\mathrm{~km} \mathrm{~s}^{-1}\right) \\
(9)\end{array}$ & $\begin{array}{c}F \\
\left(\text { Jy km s }^{-1}\right) \\
(10)\end{array}$ & $\begin{array}{l}\mathrm{S} / \mathrm{N} \\
(11)\end{array}$ & $\begin{array}{c}\log M_{\mathrm{HI}} \\
\left(\mathrm{M}_{\odot}\right) \\
(12)\end{array}$ \\
\hline 181518 & J082522.13+325953.6 & $0.1711(2)$ & -23.45 & 65 & 112 & 0.1710 & $515 \pm 23$ & 417 & $0.26 \pm 0.05$ & 8.0 & 10.54 \\
\hline 252580 & J151337.28+041921.1 & $0.1754(2)$ & -23.33 & 64 & 84 & 0.1750 & $478 \pm 08$ & 384 & $0.32 \pm 0.06$ & 8.4 & 10.65 \\
\hline 262033 & $\mathrm{~J} 162515.41+280530.3$ & $0.1870(2)$ & -23.90 & 76 & 260 & 0.1871 & $643 \pm 43$ & 518 & $0.24 \pm 0.03$ & 9.4 & 10.58 \\
\hline 224321 & $\mathrm{~J} 120948.14+100822.5$ & $0.1879(2)$ & -23.56 & 46 & 80 & 0.1875 & $417 \pm 14$ & 328 & $0.26 \pm 0.05$ & 8.9 & 10.63 \\
\hline 101750 & J003610.70+142246.4 & $0.1909(1)$ & -23.67 & 62 & 156 & 0.1909 & $542 \pm 21$ & 432 & $0.19 \pm 0.03$ & 8.2 & 10.51 \\
\hline 242091 & J140522.72+052814.6 & $0.1954(2)$ & -24.00 & 63 & 156 & 0.1953 & $502 \pm 04$ & 397 & $0.25 \pm 0.04$ & 10.3 & 10.64 \\
\hline 232055 & $\mathrm{~J} 134211.36+053211.5$ & $0.2031(1)$ & -23.45 & 57 & 92 & 0.2030 & $464 \pm 10$ & 362 & $0.26 \pm 0.05$ & 8.4 & 10.69 \\
\hline 262029 & $\mathrm{~J} 160938.00+312958.5$ & $0.2188(2)$ & -24.20 & 53 & 136 & 0.2186 & $642 \pm 56$ & 503 & $0.34 \pm 0.05$ & 9.0 & 10.88 \\
\hline 212941 & $\mathrm{~J} 111645.15+054210.0$ & $0.2239(1)$ & -23.84 & 54 & 180 & 0.2236 & $383 \pm 39$ & 289 & $0.18 \pm 0.04$ & 7.0 & 10.62 \\
\hline 242147 & $\mathrm{~J} 142735.69+033434.2$ & $0.2454(1)$ & -24.11 & 51 & 176 & 0.2454 & $499 \pm 11$ & 377 & $0.28 \pm 0.05$ & 8.0 & 10.90 \\
\hline
\end{tabular}

